PROCEEDINGS OF THE

AMERICAN MATHEMATICAL SOCIETY

Volume 135, Number 6, June 2007, Pages 1763-1767

S 0002-9939(06)08667-9

Article electronically published on November 7, 2006

\title{
OPERATORS THAT ADMIT A MOMENT SEQUENCE, II
}

\author{
B. ChevreAU, I. B. JUNG, E. KO, AND C. PEARCY \\ (Communicated by N. Tomczak-Jaegermann)
}

\begin{abstract}
As the title indicates, this note is a continuation of a paper by Foias, Jung, Ko and Pearcy, in which it was shown that certain classes of operators on a Hilbert space admit moment sequences. Herein we extend these results.
\end{abstract}

\section{INTRODUCTION}

In this note $\mathcal{H}$ will always be a separable, infinite-dimensional, complex Hilbert space, and $\mathcal{L}(\mathcal{H})$ will denote the algebra of all bounded linear operators on $\mathcal{H}$. As usual, $\mathbf{K}=\mathbf{K}(\mathcal{H})$ will denote the ideal of compact operators in $\mathcal{L}(\mathcal{H})$, and we write $\mathbb{N}\left[\mathbb{N}_{0}\right.$ ] for the set of positive [nonnegative] integers. Following [1] and [7], we say that an operator $T$ in $\mathcal{L}(\mathcal{H})$ admits a moment sequence if there exist nonzero vectors $x$ and $y$ in $\mathcal{H}$ and a (finite, regular) Borel measure $\mu$ supported on the spectrum $\sigma(T)$ of $T$ such that for every complex polynomial $p$,

$$
\langle p(T) x, y\rangle=\int_{\sigma(T)} p(\lambda) d \mu(\lambda) .
$$

(We use the term measure here in the usual sense of a nonnegative-valued set function.)

The motivation for [7] (and this continuation) is the following nice theorem of Atzmon and Godefroy [1].

Theorem 1.1. Suppose $\mathcal{X}$ is a real separable Banach space and $T$ is a bounded linear operator on $\mathcal{X}$ that admits a moment sequence (with associated Borel measure $\mu$ supported on $\sigma(T) \subset \mathbb{R}$ ). Then $T$ has a nontrivial invariant subspace.

It is obvious that every $T$ in $\mathcal{L}(\mathcal{H})$ that has a nontrivial invariant subspace (n.i.s.) admits a moment sequence (associated with the measure $\mu \equiv 0$ on $\sigma(T)$ ), and Theorem 1.1 points in the direction of the possible equivalence of the two concepts. Thus the authors believe that the question of which operators in $\mathcal{L}(\mathcal{H})$ can be shown to have a moment sequence is worth further exploration.

The basic tool used in [7] was a rather deep theorem of Foias-Pasnicu-Voiculescu [8, together with the theory of quasitriangular operators (cf., e.g., [5]), and its main theorem was the following, where $(\mathbf{N}+\mathbf{K})$ denotes the set of all operators $T$

Received by the editors January 9, 2006 and, in revised form, January 31, 2006.

2000 Mathematics Subject Classification. Primary 47A15, 44A60; Secondary 47B20.

Key words and phrases. Moment sequence, invariant subspace, hyponormal operator.

This work was supported by Korea Research Foundation Grant KRF-2002-070-C00006.

(C)2006 American Mathematical Society Reverts to public domain 28 years from publication 
in $\mathcal{L}(\mathcal{H})$ that can be written as a sum $T=N+K$, where $N$ is a normal operator and $K$ is compact.

Theorem $1.2([7)$. Every $T \in(\mathbf{N}+\mathbf{K})$ admits a moment sequence.

In this note we first show that Theorem 1.2 has a somewhat shorter proof that can be based on a theorem of Lomonosov [6], and then we modestly enlarge the class of operators known to admit moment sequences.

\section{A NEW PROOF}

We write, as usual, $\pi: \mathcal{L}(\mathcal{H}) \rightarrow \mathcal{L}(\mathcal{H}) / \mathbf{K}$ for the Calkin map, and $\sigma_{e}(T):=$ $\sigma(\pi(T)),\|T\|_{e}:=\|\pi(T)\|$. The above-mentioned result of Lomonosov is the following.

Theorem 2.1 ([6]). Let $\mathcal{A}$ be a proper $($ i.e., $\mathcal{A} \neq \mathcal{L}(\mathcal{H})$ ) subalgebra of $\mathcal{L}(\mathcal{H})$ that is closed in the weak operator topology (WOT) and contains the identity operator $1_{\mathcal{H}}$. Then there exist nonzero vectors $x$ and $y$ in $\mathcal{H}$ such that

1) $\langle x, y\rangle \geq 0$, and

2) the linear functional $\varphi \in \mathcal{A}^{*}$ defined by $\varphi(A)=\langle A x, y\rangle$ satisfies $|\varphi(A)| \leq$ $\langle x, y\rangle\|A\|_{e}$ for every $A \in \mathcal{A}$ (and therefore also satisfies $\varphi\left(1_{\mathcal{H}}\right)=\langle x, y\rangle=\|\varphi\|$ ).

Following [6], we say that a functional $\varphi \in \mathcal{A}^{*}$ with the above properties is a positive, vector functional on $\mathcal{A}$. Recall that an operator $T$ in $\mathcal{L}(\mathcal{H})$ is called essentially normal if $T^{*} T-T T^{*} \in \mathbf{K}$, i.e., if $\pi(T)$ is normal. We begin our program with the following new proof of Theorem 1.2.

New proof of Theorem 1.2. Let $\mathcal{A}_{T}$ be the unital, WOT-closed subalgebra of $\mathcal{L}(\mathcal{H})$ generated by $T$. Since $\mathcal{A}_{T}$ is abelian, $\mathcal{A}_{T} \neq \mathcal{L}(\mathcal{H})$. We apply Theorem 2.1 to obtain nonzero vectors $x, y$ in $\mathcal{H}$ and the positive vector functional $\varphi$ on $\mathcal{A}_{T}$ satisfying $\varphi(A)=\langle A x, y\rangle$ and $|\varphi(A)| \leq\langle x, y\rangle\|A\|_{e}$ for every $A \in \mathcal{A}_{T}$. Then, as above, $\varphi\left(1_{\mathcal{H}}\right)=\langle x, y\rangle=\|\varphi\|$, and we also have $\varphi\left(\mathcal{A}_{T} \cap \mathbf{K}\right)=0$. Thus, by standard facts about factoring through quotient algebras, there exists $\widehat{\varphi} \in\left(\pi\left(\mathcal{A}_{T}\right)\right)^{*}$ such that $\varphi=\widehat{\varphi} \circ \pi$ and $\widehat{\varphi}\left(\pi\left(1_{\mathcal{H}}\right)\right)=\langle x, y\rangle=\|\widehat{\varphi}\|$. In particular, we have, for every (complex) polynomial $p$,

$$
\varphi(p(T))=\widehat{\varphi}(\pi(p(T)))=\widehat{\varphi}(p(\pi(T))) .
$$

From the hypothesis, we know that $\pi(T)$ is normal, and writing $\mathcal{P}(\pi(T))$ for the polynomial algebra generated by $\pi(T)$, the Hahn-Banach theorem yields the fact that $\left.\hat{\varphi}\right|_{\mathcal{P}(\pi(T))}$ has an extension $\widehat{\varphi}_{\text {ext }}$ to the abelian unital $C^{*}$-algebra $C^{*}(\pi(T))$ generated by $\pi(T)$ satisfying

$$
\left\|\widehat{\varphi}_{\mathrm{ext}}\right\|=\left\|\widehat{\varphi}_{\mathrm{ext}}\left(\pi\left(1_{\mathcal{H}}\right)\right)\right\|=\langle x, y\rangle .
$$

In other words, $\widehat{\varphi}_{\text {ext }} \in\left(C^{*}(\pi(T))\right)^{*}$, and since this dual space is isometrically isomorphic to the Banach space $\mathcal{M}\left(\sigma_{e}(T)\right)$ of all complex, regular, Borel measures on $\sigma(\pi(T))=\sigma_{e}(T)$, we obtain, finally, that there exists $\mu \in \mathcal{M}\left(\sigma_{e}(T)\right)$ such that for every complex polynomial $p$,

$$
\langle p(T) x, y\rangle=\varphi(p(T))=\widehat{\varphi}(\pi(p(T)))=\widehat{\varphi}_{\mathrm{ext}}(p(\pi(T)))=\int_{\sigma_{e}(T)} p(\lambda) d \mu(\lambda) .
$$

Moreover, since $\varphi, \widehat{\varphi}$, and $\widehat{\varphi}_{\text {ext }}$ are positive linear functionals, the corresponding complex measure $\mu$ is, in fact, a measure, so the proof is complete. 
Using Theorem 1.2, the BDF-theory of essentially normal operators (cf. [3]), and the characterization of quasitriangular operators from [2], as well as the BergerShaw theorem [4, we now obtain, as in [7], the following.

Corollary 2.2 ([7]). Every $T$ in $\mathcal{L}(\mathcal{H})$ that is either nonbiquasitriangular, essentially normal, or hyponormal admits a moment sequence.

\section{Some NeW Results}

In this section we enlarge the class of operators in $\mathcal{L}(\mathcal{H})$ known to have a moment sequence. We first recall from [9] that an operator $T$ in $\mathcal{L}(\mathcal{H})$ is called almost hyponormal if $T^{*} T-T T^{*}$ can be written as $P+K$, where $P \geq 0$ and $K \in \mathcal{C}_{1}(\mathcal{H})$, the ideal of trace-class operators in $\mathcal{L}(\mathcal{H})$. A little-known theorem from [9] is the following.

Theorem $3.1(9])$. Suppose $T \in \mathcal{L}(\mathcal{H})$ is almost hyponormal, and let $X$ be any Hilbert-Schmidt operator in $\mathcal{L}(\mathcal{H})$ (i.e., $X \in \mathcal{C}_{2}(\mathcal{H})$ ). Then, if $T^{*} T-T T^{*} \notin \mathcal{C}_{1}(\mathcal{H})$, the operator $T+X$ has an n.i.s.

Our first new result partially generalizes Corollary 2.2.

Theorem 3.2. Every operator in $\mathcal{L}(\mathcal{H})$ of the form $T+X$, where $T$ is almost hyponormal and $X \in \mathcal{C}_{2}(\mathcal{H})$, admits a moment sequence.

Proof. If $T^{*} T-T T^{*} \notin \mathcal{C}_{1}(\mathcal{H})$, then by Theorem 3.1, $T+X$ has an n.i.s. and thus admits a moment sequence. On the other hand, if $T^{*} T-T T^{*} \in \mathcal{C}_{1}(\mathcal{H})$, then $T+X$ is essentially normal, and thus admits a moment sequence via Corollary 2.2 .

Our second new result shows that, indeed, the properties of having an n.i.s. and admitting a moment sequence are equivalent for a very special class of operators.

Theorem 3.3. Suppose $T \in \mathcal{L}(\mathcal{H})$ and $\sigma(T)$ contains at least one isolated point. Then $T$ has an n.i.s. if and only if $T$ admits a moment sequence.

Proof. If $\sigma(T)$ is not a singleton, then $\sigma(T)$ is disconnected, and hence $T$ has an n.i.s. and admits a moment sequence. The case remaining is that in which $\sigma(T)=\left\{\lambda_{0}\right\}$ for some $\lambda_{0} \in \mathbb{C}$. Note first that if $\lambda_{0}=0$ and $T$ admits a moment sequence, say

$$
\left\langle T^{n} x, y\right\rangle=\int_{\left\{\lambda_{0}\right\}} \lambda^{n} d \mu, \quad n \in \mathbb{N}_{0},
$$

for some nonzero vectors $x$ and $y$ and some (necessarily atomic) measure $\mu$ supported on $\{0\}$, then $\left\langle T^{n} x, y\right\rangle=0$ for $n \in \mathbb{N}$, and the vector $T x$ is not cyclic for $T$. Thus

$$
\mathcal{M}=\bigvee_{n \in \mathbb{N}} T^{n} x
$$

is an n.i.s. for $T$. Therefore to complete the proof it suffices to show that translation of an arbitrary operator $A$ in $\mathcal{L}(\mathcal{H})$ by an arbitrary scalar preserves the property of admitting a moment sequence. But this is immediate from (1.1) and the obvious fact that $\{p(A): p \in \mathbb{C}[x]\}=\left\{q\left(A+\gamma \cdot 1_{\mathcal{H}}\right): q \in \mathbb{C}[x]\right\}$ after making the appropriate change of variables.

We next recall that a corollary of the proof of Theorem 1.2 given in [7] was the following. 
Corollary 3.4. Suppose $T \in(\mathbf{N}+\mathbf{K})$ and $(0) \neq \mathcal{M}$ is an n.i.s. for $T$. Then $\left.T\right|_{\mathcal{M}}$ admits a moment sequence.

It is worthwile to consider the structure of $\left.\operatorname{such} T\right|_{\mathcal{M}}$. If $\operatorname{dim} \mathcal{M}=n \in \mathbb{N}$, then $\left.T\right|_{\mathcal{M}}$ is essentially an $n \times n$ complex matrix, and nothing more need be said. On the other hand, if $\operatorname{dim}(\mathcal{H} \ominus \mathcal{M}) \in \mathbb{N}$, then a matricial calculation and use of the well-known fact that $T \in(\mathbf{N}+\mathbf{K})$ if and only if $T$ is essentially normal and biquasitriangular yields the fact that $\left.T\right|_{\mathcal{M}} \in(\mathbf{N}+\mathbf{K})(\mathcal{M})$. Thus we now consider the structure of $\left.T\right|_{\mathcal{M}}$ when both $\mathcal{M}$ and $\mathcal{H} \ominus \mathcal{M}$ are infinite dimensional. Upon identifying $\mathcal{H} \ominus \mathcal{M}$ with $\mathcal{M}$, we may suppose that $T \in(\mathbf{N}+\mathbf{K})(\mathcal{M} \oplus \mathcal{M})$, and thus may be written matricially as

$$
T=\left(\begin{array}{cc}
T_{11} & T_{12} \\
0 & T_{22}
\end{array}\right)
$$

where the $T_{i j} \in \mathcal{L}(\mathcal{M})$. Let us write $T=N+K$ with $N$ normal and $K \in \mathbf{K}$, and write the corresponding $2 \times 2$ matrices for $N$ and $K$ as

$$
N=\left(\begin{array}{ll}
N_{11} & N_{12} \\
N_{21} & N_{22}
\end{array}\right), \quad K=\left(\begin{array}{ll}
K_{11} & K_{12} \\
K_{21} & K_{22}
\end{array}\right),
$$

where again the $N_{i j}$ and $K_{i j} \in \mathcal{L}(\mathcal{M})$. Obviously, $K_{i j} \in \mathbf{K}(\mathcal{M})$ for $i, j=1,2$ and $N_{21}=-K_{21} \in \mathbf{K}$. Thus $\pi_{\mathcal{M}}\left(N_{21}\right)=0, \pi_{\mathcal{M} \oplus \mathcal{M}}(T)$ is normal, and $\pi_{\mathcal{M}}\left(T_{11}\right)$ is subnormal. Since $T_{11}=\left.T\right|_{\mathcal{M}}$ has a moment sequence, some essentially subnormal operators have moment sequences. This raises the following interesting problem.

Problem 3.5. Does every essentially subnormal or essentially hyponormal operator in $\mathcal{L}(\mathcal{H})$ admit a moment sequence?

Theorem 3.2 above gives a partial answer to this question, and this next proposition gives another.

Proposition 3.6. Every $T=S+K \in \mathcal{L}(\mathcal{H})$ with $S$ subnormal and $K \in \mathbf{K}$ has a moment sequence.

Proof. Let $N$ be a minimal normal extension of $S$ acting on a Hilbert space $\mathcal{K} \supset \mathcal{H}$ and let $J=K \oplus 0_{\mathcal{K} \ominus \mathcal{H}}$ be a (compact) extension of $K$. Then clearly $N+J \in$ $(\mathbf{N}+\mathbf{K})(\mathcal{K}),(N+J) \mathcal{H} \subset \mathcal{H}$, and $\left.(N+J)\right|_{\mathcal{H}}=S+K$. So the result follows from Corollary 3.4.

We close this note with some additional interesting problems in this area.

Problem 3.7. Let $T$ be an invertible operator in $\mathcal{L}(\mathcal{H})$ admitting a moment sequence. Does $T^{-1}$ admit a moment sequence?

Problem 3.8. Suppose that for $n \in \mathbb{N}, T_{n} \in \mathcal{L}(\mathcal{H})$ admits a moment sequence and $\left\|T_{n}-T_{0}\right\| \rightarrow 0$. Does $T_{0}$ admit a moment sequence?

Problem 3.9. Does every quasinilpotent operator admit a moment sequence? In this connection, remember Theorem 3.3.

\section{REFERENCES}

[1] A. Atzmon and G. Godefroy, An application of the smooth variational principle to the existence of nontrivial invariant subspaces, C.R. Acad. Sci. Paris Sér. I Math. 332(2001), 151-156. MR.1813773(2002a:47006)

[2] C. Apostol, C. Foias and D. Voiculescu, Some results on non-quasitriangular operators, IV, Rev. Roumaine Math. Pures Appl. 18(1973), 487-514. MR0333785 (48:12109a) 
[3] L. Brown, R.G. Douglas, and P. Fillmore, Extensions of $C^{*}$-algebras and K-homology, Ann. of Math. 105(1977), 265-324. MR0458196 (56:16399)

[4] C. Berger and B. Shaw, Selfcommutators of multicyclic hyponormal operators are trace class, Bull. Amer. Math. Soc. 79(1973), 1193-1199. MR0374972 (51:11168)

[5] R. Douglas and C. Pearcy, A note on quasitriangular operators, Duke Math. J. 37(1970), 177-188. MR 0257790(41:2439)

[6] V. Lomonosov, Positive functionals on general operator algebras, J. Math. Anal. Appl. 245(2000), 221-224. MR1756586 (2000m:47098)

[7] C. Foias, I. Jung, E. Ko and C. Pearcy, Operators that admit a moment sequence, Israel J. Math. 145(2005), 83-91. MR2154721 (2006c:47010)

[8] C. Foias, C. Pasnicu, and D. Voiculescu, Weak limits of almost invariant projections, J. Operator Theory 2(1979), 79-93. MR0553865 (81m:47012)

[9] D. Voiculescu, A note on quasitriangularity and trace-class self-commutators, Acta Sci. Math. (Szeged) 42(1980), 1303-1320. MR0576955 (81k:47035)

UFR de Mathématiques et d'Informatique, Université de Bordeaux I, 351, Cours de la Libération, 33405 Talence, France

E-mail address: bernard.chevreau@math.u-bordeaux.fr

Department of Mathematics, College of Natural Science, Kyungpook National UniVERSity, DAEGu 702-701, Korea

E-mail address: ibjung@knu.ac.kr

Department of Mathematics, Ewha Women's University, Seoul 120-750, Korea

E-mail address: eiko@ewha.ac.kr

Department of Mathematics, Texas A\&M University, College Station, Texas 77843

E-mail address: pearcy@math.tamu.edu 